\title{
Distinct roles for dorsal CA3 and CA1 in memory for sequential nonspatial events
}

\author{
Anja Farovik, Laura M. Dupont, and Howard Eichenbaum ${ }^{1}$ \\ Center for Memory and Brain, Boston University, Boston, Massachusetts 02215, USA
}

\begin{abstract}
Previous studies have suggested that dorsal hippocampal areas $C A 3$ and $C A 1$ are both involved in representing sequences of events that compose unique episodes. However, it is uncertain whether the contribution of $C A 3$ is restricted to spatial information, and it is unclear whether CAl encodes order per se or contributes by an active maintenance of memories of sequential events. Here, we developed a new behavioral task that examines memory for the order of sequential nonspatial events presented as trial-unique odor pairings. When the interval between odors within a studied pair was brief $(3 \mathrm{sec})$, bilateral dorsal CA3 lesions severely disrupted memory for their order, whereas dorsal CA1 lesions did not affect performance. However, when the inter-item interval was extended to $10 \mathrm{sec}$, CAl lesions, as well as CA3 lesions, severely disrupted performance. These findings suggest that the role of $C A 3$ in sequence memory is not limited to spatial information, but rather appears to be a fundamental property of CA3 function. In contrast, CA1 becomes involved when memories for events must be held or sequenced over long intervals. Thus, CA3 and CAl are both involved in memory for sequential nonspatial events that compose unique experiences, and these areas play different roles that are distinguished by the duration of time that must be bridged between key events.
\end{abstract}

Episodic memory involves the ability to encode and retrieve the order of events in individual experiences (Tulving 1983). Recent evidence in both animals and humans indicates that the hippocampus plays a critical role in this capacity. In animals, damage to the hippocampus impairs memory for the order of associated elements that compose an episode (Fortin et al. 2002; Kesner et al. 2002), and hippocampal neuronal activity reflects processing of the order of events in both spatial (Dragoi and Buzsáki 2006; Foster and Wilson 2007) and nonspatial episodes (Manns et al. 2007). In humans, hippocampal activation has also been related to memory for the order of elements (Kumaran and Maguire 2006; Lehn et al. 2009; Ross et al. 2009).

Within the hippocampal circuitry, contributions of the CA3 and CA1 fields are probably most extensively studied, but this work has not yet clarified the distinct roles of these areas in sequence memory. Computational models suggest that the recurrent connections of CA3 cells operate as an attractor network that computes associations between elements (Norman and O'Reilly 2003; Rolls 2007) and is suitable for representing sequences of events in episodic memories (Jensen and Lisman 1996; Levy 1996; Lisman 1999). Studies on the effects of selective damage within the hippocampus have shown that CA3 is critical for remembering sequences of spatial locations (Hunsaker et al. 2008a), but not sequences of nonspatial events (Hoge and Kesner 2007). It is, therefore, uncertain whether CA3 is critical for sequence memory per se, rather than other aspects of spatial processing. Other observations suggest that CA1 may be involved in memory for the order of both spatial (Hunsaker et al. 2008a) and nonspatial stimuli (Hoge and Kesner 2007; Manns et al. 2007). However, it is not clear whether the contribution of CA1 involves integrating sequential elements of a memory or instead participates by active maintenance of event memories that underlies bridging sequential events in an episode (Kesner et al. 2005).

To shed light on these issues, we compared the effects of selective damage to CA 3 and CA 1 on memory for the order of

\footnotetext{
'Corresponding author.
}

E-mail hbe@bu.edu; fax (617) 353-1414.

Article is online at http://www.learnmem.org/cgi/doi/10.1101//m.1616209. nonspatial events that occurred in unique episodes. We designed a task, based on the delayed-nonmatching-to-sample test, wherein subjects were required to remember the order of two sequentially presented stimuli in trial-unique-paired associations (Fig. 1).

\section{Results}

\section{Preoperative performance}

In each daily session, animals studied 10 odor pairs, followed by a 10-min delay, and subsequently a test phase. At test, animals were required to distinguish test pairs where the order of odor presentation within a pair was the same as that during study ("old" order) from test pairs where the order of odor presentation was reversed ("new" order). A one-way ANOVA with group (control, CA1, and CA3) as the single between-subjects factor and percent correct as the dependent variable indicated that the groups $(n=6$ for each group) were well matched on performance prior to surgery $\left(F_{(2,15)}=0.38\right.$, ns) (Fig. $4 \mathrm{~A}$, see below). In addition, we also compared the proportion of errors of omission (incorrect responses to "new" order test pairs) relative to the total number of response errors (old + new order test pairs). This analysis indicated that each group produced a similar response error pattern $\left(F_{(2,15)}=\right.$ 0.014 , ns). Subsequently, each group received bilateral ibotenic lesions of the dorsal CA3 region, dorsal CA1 region, or control saline injections.

\section{Anatomical observations}

Figure 2 shows the area of damage at the three injection sites relative to bregma (photomicrographs of a control and animals with lesions of dorsal CA3 and CA1 are provided in Fig. 3). Histological quantification was carried out using Canvas 7. A calculation of the total area of dorsal CA3 and CA1 was conducted for the region extending from $\mathrm{AP}-2.12$ to $\mathrm{AP}-5.16$. Then, the area of the lesion within each subdivision was measured and the proportion of subdivision affected by the lesion was estimated. In rats with dorsal CA3 lesions, histological quantification revealed bilateral damage to area CA3 that extended from the dentate gyrus to the borders of CA2. On average, the area damaged within CA3 was $66 \%$ at $-2.80 \mathrm{~mm}, 58 \%$ at $-3.80 \mathrm{~mm}$, and $61 \%$ at $-4.30 \mathrm{~mm}$ 


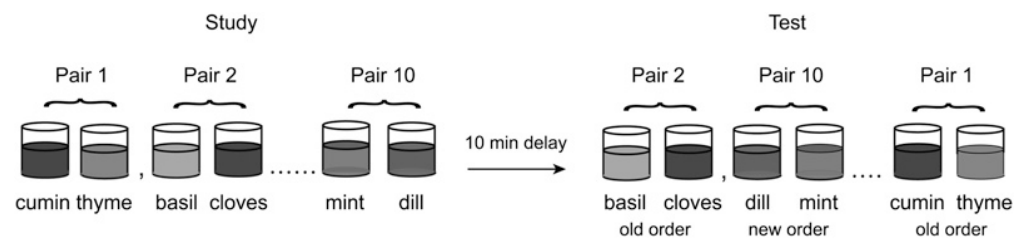

Figure 1. Test of memory for the order of stimuli in trial-unique odor pairs. At study, animals were presented with 10 odor-paired associates and odors in a pair were presented one at a time. At test, animals were presented with the same 10 odor pairs and were required to distinguish pairs where the odors within a pair were presented in the same order as during study ("old") from pairs where the odors were presented in the reverse order ("new"). Old and new order test pairs were presented in a pseudorandom order. The first odor in each test pair acted as a cue to the ordering of the odors within a test pair; the animal was required to place its nose over the cup, but no digging response was required or rewarded. When the second cup was presented, the animal could dig to retrieve a reward if the order was new. If the order was old, the animal was required to approach an empty cup in the back of the home cage to obtain reward.

posterior to bregma. Across these three injection sites the overall average damage was $62 \%$. One animal exhibited minor damage to the dorsal lateral geniculate and slight damage to dorsal CA1 that borders CA2. Some damage was also evident to the dorsal region of the fimbria in two animals, and was common to CA2 and parts of the dentate gyrus.

In animals with lesions of dorsal CA1, the damage estimate was carried out from the fasciola cinereum (FC) to CA2. On average, the loss in CA1 was $46 \%$ at $-2.80 \mathrm{~mm}, 42 \%$ at $-3.80 \mathrm{~mm}$, and $29 \%$ at $-4.30 \mathrm{~mm}$ posterior to bregma. Across these three injection sites, the overall average CA1 damage was 39\%. One animal had minor damage quite anterior, but damage was uncommon at anterior coordinates beyond approximately $-2.52 \mathrm{~mm}$. In two animals, minor damage to the dentate gyrus and CA2 was evident, and slight damage in area CA3 that borders CA2. Lastly, slight damage to the corpus callosum and dorsal subiculum, more posteriorly, was common in CA1-lesioned animals, and one rat exhibited partial damage in the dorsal fornix. Although the estimated mean percent lesion size was larger for CA3 than CA1, this difference was not statistically significant $\left(t_{(10)}=-1.4, P=\right.$ $0.19, \mathrm{~ns})$, nor was there a significant group difference in absolute lesion size $\left(t_{(10)}=-1.98, P=0.08, \mathrm{~ns}\right)$, due to the high variability of lesion sizes (see Fig. 2).

\section{Postoperative performance}

\section{3-sec inter-item interval}

After surgery, the performance of the three groups was compared on overall accuracy (percent correct) across the first 10 postoperative sessions. Control subjects continued to perform at a high level of accuracy (78.7\% correct). Rats with CA3 lesions were severely impaired in order memory (53.7\% correct), but CA1 rats performed as well as controls (78.3\% correct; Fig. 4B). A one-way ANOVA revealed a significant effect of lesion on accuracy $\left(F_{(2,15)}=\right.$ $33.89, P<0.001)$. Further analyses using Tukey HSD post-hoc comparison revealed that CA3 rats were impaired compared to controls $(P<0.001)$ and to CA1 rats $(P<0.001)$; and CA3 rats did not perform better than chance $\left(t_{(5)}=1.40\right.$, ns). In contrast, the performance of rats with CA1 lesions did not differ from that of controls $(P>0.05, \mathrm{~ns})$. The proportion of errors of omission did not differ among groups $\left(F_{(2,15)}=0.014, n s\right)$, indicating that the severe memory impairment in CA3 rats cannot be attributed to a shift in the general response pattern.

\section{Inter-item interval prolonged}

\section{0-sec inter-item interval}

After assessment of postoperative performance using an inter-item interval of $3 \mathrm{sec}$ within each studied paired associate, the inter- item interval was extended to $10 \mathrm{sec}$ to examine the extent to which an intact hippocampal area is necessary for recognition memory of item order when temporal processing demands increase. At test, the inter-item interval remained at 3 sec. Again, control subjects performed at a high level of accuracy (76\% correct), and rats with $\mathrm{CA} 3$ lesions continued to exhibit severe impairment in order memory $(53 \%$ correct). In contrast to performance with the 3-sec inter-item interval, animals with CA1 lesions displayed impaired order memory ( $57 \%$ correct) at the 10-sec inter-item interval (Fig. 4C). A one-way ANOVA was performed on overall percent correct across six sessions that contained a 10-sec inter-item interval between odors in each pair at study. Analysis continued to show a group difference in performance accuracy $\left(F_{(2,15)}=24.32, P<0.001\right)$, and post-hoc group comparisons revealed a different pattern than that with the 3 -sec inter-item interval. In particular, at the 10 -sec inter-item interval, rats with CA1 lesions were severely impaired compared to controls $(P<0.001)$, although the performance of CA1 rats did exceed that expected by chance $\left(t_{(5)}=2.66, P<0.05\right)$. Animals with lesions to CA3 continued to exhibit a performance deficit compared to controls $(P<0.001)$, and comparison between CA1 and CA3 groups revealed comparable levels of accuracy when the inter-item interval was prolonged $(P=0.53$, ns). Additional analysis revealed that the poor performance by both CA3- and CA1-lesioned animals could not be attributed to a shift in the general response pattern $\left(F_{(2,15)}=0.024, \mathrm{~ns}\right)$.

To explore whether the difference in the patterns of performance between inter-item intervals could be attributed to increased task difficulty at the longer interval, we compared the performance of control rats at the two intervals. A paired $t$-test analysis revealed that the extended 10 -sec inter-item interval did not significantly increase task difficulty compared to the 3-sec inter-item interval for control subjects $\left(t_{(5)}=1.26\right.$, ns). Nevertheless, the performance of CA1 rats was significantly reduced compared to their performance at the 3 -sec inter-item interval $\left(t_{(5)}=18.8, P<0.001\right)$.

\section{Discussion}

This study examined the role of dorsal hippocampal subregions CA3 and CA1 in memory for the order of sequentially associated nonspatial elements. Consistent with the observation that the hippocampus participates in sequence memory (Fortin et al. 2002; Kesner et al. 2002; Lehn et al. 2009; Ross et al. 2009), we observed that memory was severely impaired after selective dorsal CA3 and CA1 damage. In addition, a subregional analysis revealed that the pattern of deficit differed between the groups with selective CA3 and CA1 lesions. Whereas memory was severely affected by damage to dorsal CA3 even when temporal processing demands were minimal, dorsal CA1 damage produced a memory impairment only when the temporal processing demands were increased. The deficit in both groups could not be explained by a shift in the general response patterns.

Our observation that memory was severely affected by CA3 lesions supports models that propose that CA3 operates as an associative memory network suitable for representing sequences of events (Jensen and Lisman 1996; Levy 1996; Lisman 1999), and indicates that the attractor dynamics of CA3 architecture provide a general mechanism by which associations between elements can be formed and stored in memory. Moreover, our results are in 


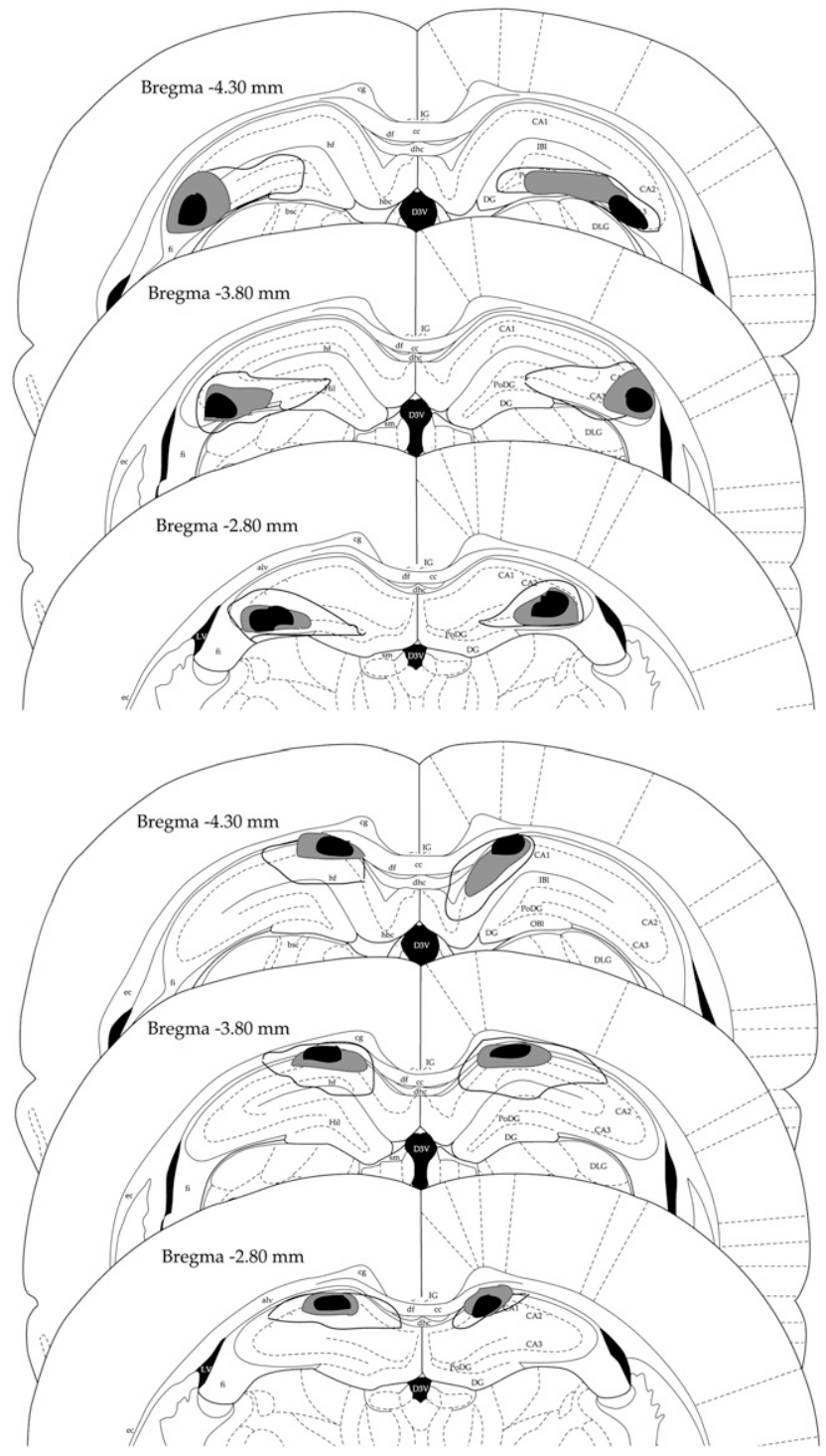

Figure 2. Reconstruction of lesions to dorsal hippocampal subregions CA3 (top) and CA1 (bottom) at $-2.80,-3.80$, and $-4.30 \mathrm{~mm}$ posterior to bregma. Black, smallest lesion; light gray, average lesion across animals; solid line, largest lesion.

accordance with evidence that demonstrate the importance of this structure in the rapid acquisition of new information (Nakazawa et al. 2003; Leutgeb et al. 2006; Miyashita et al. 2009). In our task, the odor combination in a paired associate is session unique (see Materials and Methods). A failure to organize and flexibly use new incoming information may underlie the memory impairment in animals with CA3 damage. This region may normally contribute to memory for unique sequences by enabling the rapid formation and storage of associations between memory cues, an ability that might be subserved by the regulation of $\mathrm{N}$-methylD-aspartate (NMDA) receptor-dependent long-term potentiation (LTP) (Bliss and Collingridge 1993; Morris and Frey 1997; Morris et al. 2003).

Another complementary account of our findings is that CA3 participates in pattern separation of experiences (Leutgeb et al. 2004; Vazdarjanova and Guzowski 2004). The hippocampus has been reported to play a role in the disambiguation of overlapping sequences, where some elements are common to two separate events (Agster et al. 2002; Kumaran and Maguire 2006). In our task, odor pairings are recombined each day. Thus, odor A may be paired with odor B on one day, but with item $C$ the following day. Even within each session, a central demand of this task is to distinguish a remembered order of stimuli within each pair from the reversed order of the same stimuli in a test pair. Accordingly, a failure to distinguish memory representations with highly overlapping patterns could account for the deficit observed here. Our results, however, cannot exclude the possibility that the impairment seen in CA3 animals was caused by a deficit in memory for individual odor "items." Nonetheless, item recognition is typically preserved after complete hippocampal damage at comparable delays (Dudchenko et al. 2000; Mumby 2001; Fortin et al. 2002), suggesting that it is unlikely that the performance in CA3 animals is caused by impaired item memory.
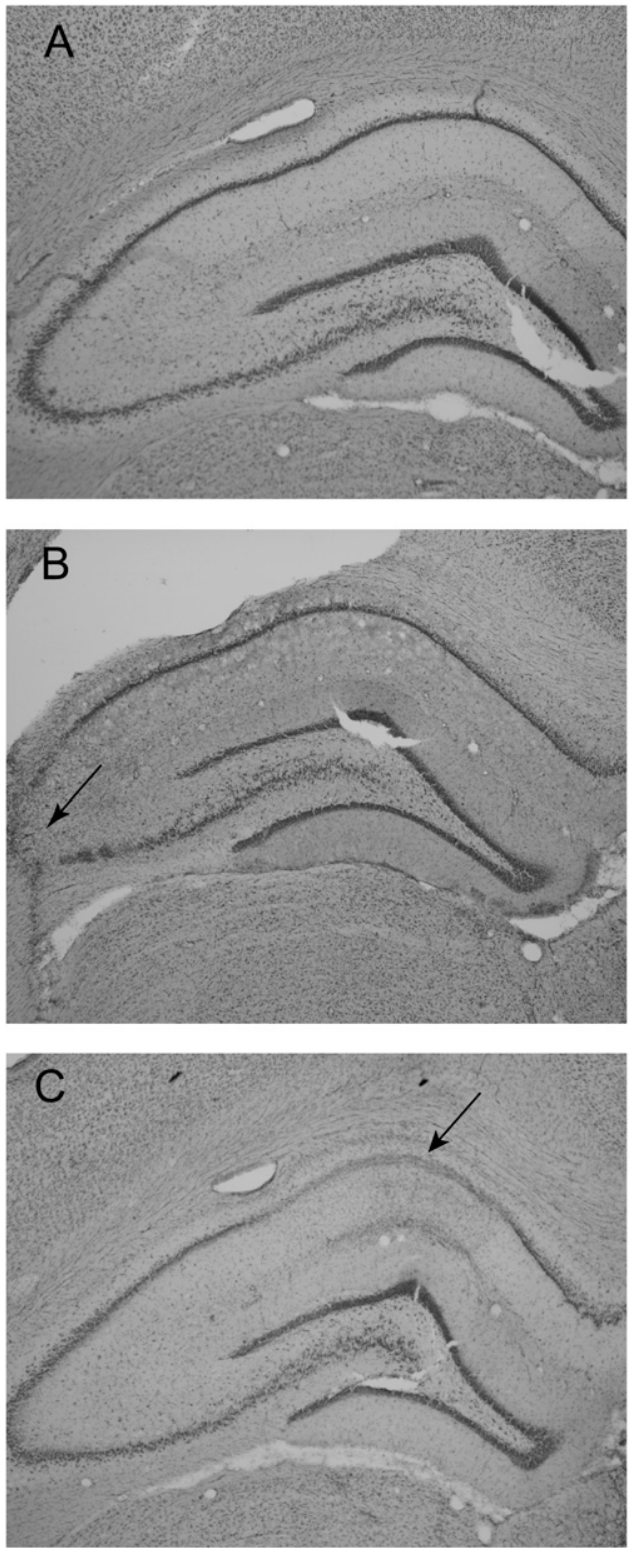

Figure 3. Photomicrographs of coronal sections stained with cresyl violet at approximately $-3.48 \mathrm{~mm}$ posterior to bregma: $(A)$ shamoperated control; (B) CA3 lesion; (C) CA1 lesion. 


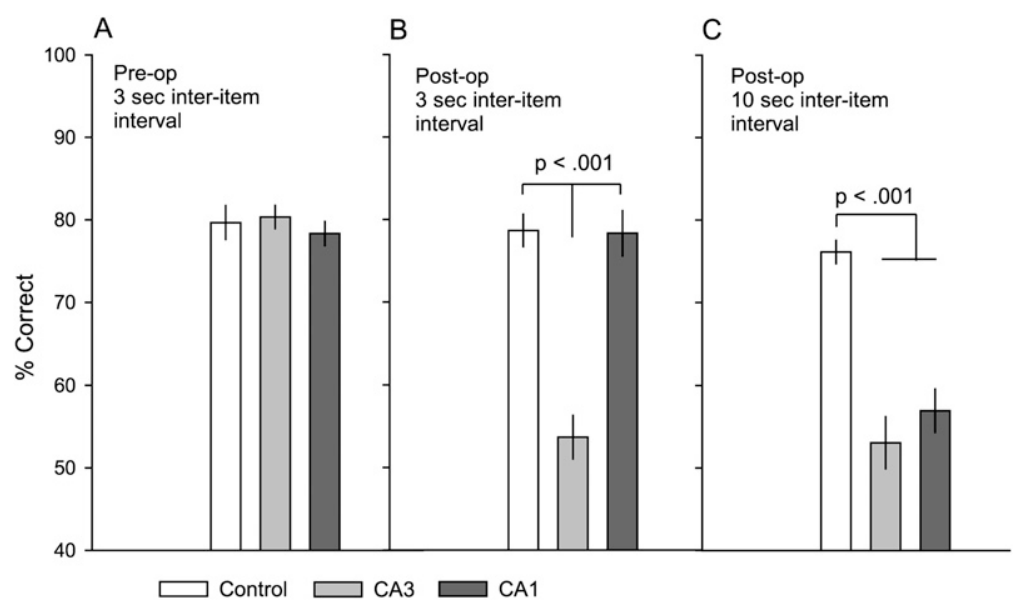

Figure 4. Memory performance in controls and rats with dorsal CA1 or CA3 lesions. (A) Percent correct ( \pm SEM) for each group in sessions prior to surgery. (B) Postoperative performance when the interval between presented odors in a pair was $3 \mathrm{sec}$ at study. Controls and rats with CA1 lesions continued to perform well, whereas rats with CA3 lesions were severely impaired. (C) Performance when the inter-item interval was extended to $10 \mathrm{sec}$ at study. Controls continued to perform well, but rats with CA1 lesions, as well as rats with CA3 lesions, were severely impaired.

Consistent with recent work that reports hippocampal involvement in binding events within experiences across time (Hoge and Kesner 2007; Manns et al. 2007; Staresina and Davachi 2009), one account of the effect of CA1 lesions in our study is that this structure makes a prominent contribution to memory encoding by binding cues to their temporal context that enables later retrieval of sequences of events. Yet, in the current study, CA1 damage did not affect memory performance when the inter-item interval at study was minimal, suggesting that order processing per se did not require CA1. Why is an intact CA1 necessary for memory of item order only when the inter-item interval exceeds a certain time frame? One possibility is that coupling within the CA3 network might be timelimited, and thus the capacity of CA3 to compute the associations becomes challenged when the inter-item interval is prolonged. CA1 might bridge an extended time gap by an, as yet undescribed, activated maintenance mechanism or by representing temporal contexts that extend over longer intervals (Manns et al. 2007).

It is possible that animals could perform the current task by encoding and remembering only the first or the second item in each paired associate. Nonetheless, if the animal did only remember one item in each pair, they would still need to label the temporally defined position of this item, i.e., is the item "first" or "last" in the pair? That is, some form of temporal processing is still required. Yet, this strategy is unlikely because if animals encoded only one of the two items in each pair, then extending the interitem interval should not have had a differential effect on memory performance. Yet, extending the inter-item interval resulted in a major reduction in performance in CA1-lesioned animals. The impairment in CA1 rats might have been driven by increased working memory and attentional demands, which leaves open the possibility that CA1 contributes to order memory by extending elements of a memory trace across time to be associated with succeeding elements. While it could be argued that a more extensive lesion of CA1 might have resulted in a deficit also at the shorter inter-item interval, our results seem consistent with previous work that observe a memory deficit after CA1 damage at similar delays (Kesner et al. 2005).

One open question is how sequence memories that are supported by CA3 at the brief inter-item interval leave the hippocampus in animals with CA1 damage, given that the major output route for CA3 within the trisynaptic circuitry is via CA1 results, however, support accumulating work that demonstrates functional differences between CA3 and CA1 (Gilbert and Kesner 2003; Daumas et al. 2005; Ji and Maren 2008), and suggest that these two hippocampal subregions play different roles in supporting memory for the order of events in unique episodes.

\section{Materials and Methods}

\section{Animals}

Subjects were 18 male Long-Evans rats (Charles River, MA) weighing between 225 and $250 \mathrm{~g}$ at the start of the experiment. All animals were single-housed and maintained on a 12-h light/ dark cycle (lights on 8:00 am to 8:00 pm). Behavioral training and testing were conducted during the light phase. Animals were kept at $\sim 85 \%$ of their free-feeding body weight and had free access to water in the home cage. Procedures were conducted according to the requirements set by the National Institutes of Health (NIH) and Boston University Institutional Animal Care and Use Committee (IACUC).

\section{Apparatus and materials}

Behavioral training and testing were carried out in the home cage $(44 \times 21 \times 20 \mathrm{~cm})$. The materials consisted of transparent Nalgene cups (VWR) $(6.5 \mathrm{~cm}$ high) with an internal diameter of $\sim 6.5 \mathrm{~cm}$. Each cup was filled with playground sand and scented with one distinct odor. A selection of 40 different odors (household spices) was used in the study. The cups were attached to a black Plexiglas platform using Velcro (VWR) before they were lowered into the cage via a wire handle. Froot Loops (Kellogg's) were used as reinforcement.

\section{Behavioral protocol}

Animals were trained in successive stages. Initially, rats were trained to dig for reward (one Froot Loop) buried in a cup filled with unscented sand. Once the animals had learned to dig reliably to retrieve the reward, they were introduced to the task. Training began with each trial consisting of one odor-paired associate. At study, the two odors in a pair were presented one at a time (e.g., A then B) with a 3-sec inter-item interval and a 10-sec study-test interval. At study, each odor in a pair was baited with a reward. At test, the same odors were either presented in the same order as during study (e.g., A then B, "old") or in the reverse order (e.g., 
B then A, "new"). Odor combinations were trial-unique. When presented with the first odor in a test pair, rats had to approach and place their nose over the cup, thus encouraging the rat to identify its odor. The first odor acted as a cue to the order of odor presentation and was never rewarded. Once the rat had placed its nose over the cup, the cup was removed and the second odor in the pair was introduced. If the order of odor presentation was the same as during study (e.g., A then B, "old"), then correct response was to refrain from digging in the second cup and approach an empty cup in the back of the home cage. If the order of presentation was reversed (e.g., B then A, "new"), then correct response was to dig in the second cup for reward. Old and new order test trials were presented in a pseudorandom order. At this stage in training, correct "new" responses were rewarded with $1 / 4$ Froot Loop buried in the cup and one whole Froot Loop was provided in the back of the cage for correct "old" responses. Trials that required the same response ("new" or "old") did not occur more than three times in a row during the test phase. Animals performed 10 trials a day.

Once the animals reached a criterion of $75 \%$ correct across 20 consecutive trials (128.6 \pm 17.03 s.d. trials to criterion), the list of studied odor pairings was increased to five per trial, and criterion was set at $75 \%$ correct across four consecutive trials $(15.7 \pm 2.44$ s.d. trials to criterion). Each pair was separated by a 15 -sec interval at both study and test, and animals completed two trials per day. To balance the presentation of "old" and "new" order test pairs at this stage of training, sessions that contained three old and two new order test pairs alternated with sessions that contained three new and two old order test pairs. Correct responses were now rewarded with $1 / 4$ Froot Loop for both response types.

Next, 10 odor pairs were studied per trial, the criterion was set to $75 \%$ correct across two consecutive trials, and the study-test interval was extended to $10 \mathrm{~min}$. At test, in five of the pairs, the odors within a pair were presented in the same order as during study (old), whereas in the other five pairings the order of odor presentation was in the reverse order (new). One trial was completed a day per animal. When animals reached criterion (15.7 \pm 2.44 s.d. trials criterion), they were divided into three matched groups based on overall percent correct across the following 10 sessions.

\section{Surgery}

Anesthesia was induced by inhalation of 5\% Isoflurane (Webster Veterinary Supply) in oxygen and was maintained at $2 \%-2.5 \%$ throughout surgery. Rats were placed in a stereotaxic frame (Kopf) and an incision was made along the midline to expose the scull. Using a 1- $\mu \mathrm{L}$ Hamilton syringe that was attached to a microsyringe pump (World Precision Instruments), six rats were given three injections per hemisphere (total of six injections) of $0.06 \mathrm{M}$ ibotenic acid (Tocris Cookson) into the hippocampal CA1 subregion at a rate of $1 \mu \mathrm{L} / \mathrm{min}$. Lesions were made using coordinates from the atlas of Paxinos and Watson (1998): from bregma, AP -2.8 $\mathrm{mm}, \mathrm{ML} \pm 1.7 \mathrm{~mm}, \mathrm{DV}-2.4 \mathrm{~mm}(0.1 \mu \mathrm{L})$ (from dura); AP -3.8 $\mathrm{mm}, \mathrm{ML} \pm 2.0 \mathrm{~mm}, \mathrm{DV}-2.0 \mathrm{~mm}(0.15 \mu \mathrm{L})$; and $\mathrm{AP}-4.3 \mathrm{~mm}$, $\mathrm{ML} \pm 2.5 \mathrm{~mm}, \mathrm{DV}-2.0 \mathrm{~mm}(0.15 \mu \mathrm{L})$. The needle was left in situ for $3 \mathrm{~min}$ after injection to allow for diffusion. Another six rats were given three injections per hemisphere (total of six injections) into hippocampal CA3 subregion at the same injection rate as for CA1 lesions: coordinates: $\mathrm{AP}-2.8 \mathrm{~mm}, \mathrm{ML} \pm 2.8 \mathrm{~mm}, \mathrm{DV}-3.3$ $\mathrm{mm}(0.1 \mu \mathrm{L}) ; \mathrm{AP}-3.8 \mathrm{~mm}, \mathrm{ML} \pm 3.9 \mathrm{~mm}, \mathrm{DV}-3.2 \mathrm{~mm}(0.15 \mu \mathrm{L})$; and $\mathrm{AP}-4.3 \mathrm{~mm}, \mathrm{ML} \pm 4.4 \mathrm{~mm}, \mathrm{DV}-3.8 \mathrm{~mm}(0.15 \mu \mathrm{L})$. Diazepam (5 mg/mL; Webster Veterinary Supply) was given intramuscularly (i.m.) immediately after surgery to prevent convulsions. Another six rats (controls) underwent the same procedure as the lesion groups but saline was infused instead of ibotenic acid. Three control animals were given saline injections at CA1 coordinates, and the other three animals had injections given at CA3 coordinates. After surgery, general health was monitored until they recovered and returned to testing, $\sim 1$ wk after surgery.

\section{Histology}

After completion of behavioral testing, rats were overdosed with $0.8 \mathrm{~mL}$ sodium pentobarbital (Fort Dodge Animal Health). Ani- mals were then perfused transcardially with $0.9 \%$ saline, followed by $10 \%$ formalin (VWR), and the brains were removed and placed in a $20 \%$ sucrose solution until processed. Using a cryostat (ReichertJung, Kramer Scientific) brains were cut into $50 \mu \mathrm{m}$ coronal sections and mounted onto pre-subbed glass slides, and stained with cresyl violet to determine the location and extent of the lesion.

\section{Acknowledgment}

This work was supported by the National Institute of Mental Health (NIMH), project grant MH52090.

\section{References}

Agster KL, Fortin NJ, Eichenbaum H. 2002. The hippocampus and disambiguation of overlapping sequences. J Neurosci 22: 5760-5768.

Amaral DG, Witter MP. 1989. The three-dimensional organization of the hippocampal formation: A review of anatomical data. Neuroscience 31: $571-591$.

Bliss TV, Collingridge GL. 1993. A synaptic model of memory: Long-term potentiation in the hippocampus. Nature 361: 31-39.

Daumas S, Halley H, Francés B, Lassalle JM. 2005. Encoding, consolidation, and retrieval of contextual memory: Differential involvement of dorsal CA3 and CA1 hippocampal subregions. Learn Mem 12: 375-382.

Dragoi G, Buzsáki G. 2006. Temporal encoding of place sequences by hippocampal cell assemblies. Neuron 50: 145-157.

Dudchenko PA, Wood ER, Eichenbaum H. 2000. Neurotoxic hippocampal lesions have no effect on odor span and little effect on odor recognition memory but produce significant impairments on spatial span, recognition, and alternation. J Neurosci 20: 2964-2977.

Fortin NJ, Agster KL, Eichenbaum HB. 2002. Critical role of the hippocampus in memory for sequences of events. Nat Neurosci 5: 458-462.

Foster DJ, Wilson MA. 2007. Hippocampal theta sequences. Hippocampus 17: $1093-1099$.

Gilbert PE, Kesner RP. 2003. Localization of function within the dorsal hippocampus: The role of the CA3 subregion in paired-associate learning. Behav Neurosci 117: 1385-1394.

Hoge J, Kesner RP. 2007. Role of CA3 and CA1 subregions of the dorsal hippocampus on temporal processing of objects. Neurobiol Learn Mem 88: $225-231$

Hunsaker MR, Lee B, Kesner RP. 2008a. Evaluating the temporal context of episodic memory: The role of CA3 and CA1. Behav Brain Res 188: 310-315.

Hunsaker MR, Tran GT, Kesner RP. 2008b. A double-dissociation of subcortical hippocampal efferents for encoding and consolidation/ retrieval of spatial information. Hippocampus 18: 699-709.

Ishizuka N, Weber J, Amaral DG. 1990. Organization of intrahippocampal projections originating from CA3 pyramidal cells in the rat. J Comp Neurol 295: 580-623.

Jensen O, Lisman JE. 1996. Hippocampal CA3 region predicts memory sequences: Accounting for the phase precession of place cells. Learn Mem 3: 279-287.

Ji J, Maren S. 2008. Differential roles for hippocampal areas CA1 and CA3 in the contextual encoding and retrieval of extinguished fear. Learn Mem 15: 244-251.

Kesner RP, Gilbert PE, Barua LA. 2002. The role of the hippocampus in memory for the temporal order of a sequences of odors. Behav Neurosci 116: $286-290$.

Kesner RP, Hunsaker MR, Gilbert PE. 2005. The role of CA1 in the acquisition of an object trace-odor paired associate task. Behav Neurosci 119: $781-786$.

Kumaran D, Maguire EA. 2006. The dynamics of hippocampal activation during encoding of overlapping sequences. Neuron 49: 617-629.

Lehn H, Steffenach HA, van Strien NM, Veltman DJ, Witter MP, Håberg AK. 2009. A specific role of the human hippocampus in recall of temporal sequences. J Neurosci 29: 3475-3484.

Leutgeb S, Leutgeb JK, Treves A, Moser MB, Moser EI. 2004. Distinct ensemble codes in hippocampal areas CA3 and CA1. Science 305: 1295-1298.

Leutgeb S, Leutgeb JK, Moser EI, Moser MB. 2006. Fast rate coding in hippocampal CA3 cell ensembles. Hippocampus 16: 765-774.

Levy WB. 1996. A sequence predicting CA3 is a flexible associator that learns and uses context to solve hippocampal-like tasks. Hippocampus 6: 579-590.

Lisman JE. 1999. Relating hippocampal circuitry to function: Recall of memory sequences by reciprocal dentate-CA3 interactions. Neuron 22: 233-242.

Manns JR, Howard MW, Eichenbaum H. 2007. Gradual changes in hippocampal activity support remembering the order of events. Neuron 56: $530-540$ 
Miyashita T, Kubik S, Haghighi N, Steward O, Guzowski JF. 2009. Rapid activation of placticity-associated gene transcription in hippocampal neurons provides a mechanism for encoding of one-trial experience. J Neurosci 29: 898-906.

Morris RG, Frey U. 1997. Hippocampal synaptic plasticity: Role in spatial learning or the automatic recording of attended experience? Philos Trans R Soc Lond B Biol Sci 352: 1489-1503.

Morris RGM, Moser EI, Riedel G, Martin SJ, Sandin J, Day M, O'Carroll C. 2003. Elements of a neurobiological theory of the hippocampus: The role of activity-dependent synaptic plasticity in memory. Philos Trans R Soc Lond B Biol Sci 358: 773-786.

Mumby DG. 2001. Perspectives on object-recognition memory following hippocampal damage: Lessons from studies in rats. Behav Brain Res 127: 159-181.

Nakazawa K, Sun LD, Quirk MC, Rondi-Reig L, Wilson MA, Tonegawa S. 2003. Hippocampal CA3 NMDA receptors are crucial for memory acquisition of one-time experience. Neuron 38: 305-315.

Norman KA, O'Reilly RC. 2003. Modeling hippocampal and neocortical contributions to recognition memory: A complementary-learningsystems approach. Psychol Rev 110: 611-646.

Paxinos G, Watson C. 1998. The rat brain in stereotaxic coordinates, 4 th ed. Academic Press, San Diego, CA.
Rolls ET. 2007. An attractor network in the hippocampus: Theory and neurophysiology. Learn Mem 14: 714-731.

Ross RS, Brown TI, Stern CE. 2009. The retrieval of learned sequences engages the hippocampus: Evidence from fMRI. Hippocampus 19: 790-799.

Staresina BP, Davachi L. 2009. Mind the gap: Binding experiences across space and time in the human hippocampus. Neuron 63: 267-276.

Swanson LW, Cowan WM. 1979. The connections of the septal region in the rat. J Comp Neurol 186: 621-655.

Treves A. 2004. Computational constraints between retrieving the past and predicting the future, and the CA3-CA1 differentiation. Hippocampus 14: $539-556$.

Tulving E. 1983. Elements of episodic memory. Clarendon, Oxford, UK.

Vazdarjanova A, Guzowski JF. 2004. Differences in hippocampal neuronal population responses to modifications of an environmental context: Evidence for distinct, yet complementary, functions of CA3 and CA1 ensembles. J Neurosci 24: 6489-6496.

Wyss X, Swanson LW, Cowan MW. 1980. The organization of the fimbria, dorsal fornix, and ventral hippocampal commissure in the rat. Anat Embryol (Berl) 158: 303-316.

Received September 2, 2009; accepted in revised form November 5, 2009. 


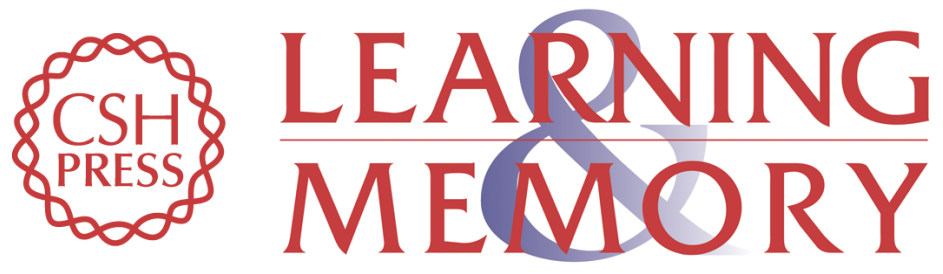

\section{Distinct roles for dorsal CA3 and CA1 in memory for sequential nonspatial events}

Anja Farovik, Laura M. Dupont and Howard Eichenbaum

Learn. Mem. 2010, 17:

Access the most recent version at doi:10.1101//m.1616209

References This article cites 37 articles, 10 of which can be accessed free at: http://learnmem.cshlp.org/content/17/1/12.full.html\#ref-list-1

License

Email Alerting Receive free email alerts when new articles cite this article - sign up in the box at the Service top right corner of the article or click here. 